\title{
Investigation of grain boundary segregation in acceptor and donor doped strontium titanate
}

\author{
N. Wilcox ${ }^{\text {a }}$, V. Ravikumar ${ }^{\text {a }}$, R.P. Rodrigues a , V.P. Dravid a, M. Vollmann ${ }^{\text {b }}$, R. Waser ${ }^{\text {b }}$, \\ K.K. Soni ${ }^{\mathrm{c}}$, A.G. Adriaens ${ }^{\mathrm{c}}$ \\ " Department of Materials Science and Engineering, Northwestern University, Evanston, IL 60208, USA \\ b Institut fur Werkstoffe der Elektrotechnik, RWTH - Aachen University of Technology, Templergraben 55, 52056 Aachen, Germany \\ ' Enrico Fermi Institute, The University of Chicago, Chicago, IL 60637, USA
}

\begin{abstract}
Grain boundary segregation in electronic ceramics is often responsible for dictating the grain boundary properties, which in turn dictate the macroscopic electronic properties of the material. Consequently, it is important to understand the nature of segregation phenomena in these materials. Here we present results from a combination of diverse analytical techniques used to investigate the character of grain boundary segregation in acceptor ( $\mathrm{Fe}, \mathrm{Mn})$ and donor $(\mathrm{Nb})$ doped strontium titanate. $\mathrm{X}$-ray emission spectroscopy (XES) and electron energy loss spectroscopy (EELS) analysis consistently show segregation of both acceptor $(\mathrm{Fe}$, and $\mathrm{Mn}$ ) and donor $(\mathrm{Nb})$ dopant species to the grain boundaries. Within the spatial resolution of the techniques, the segregation profiles for these dopants are found to be limited to less than $5 \mathrm{~nm}$ about the grain boundaries. Furthermore secondary ion mass spectroscopy shows that the segregation is ubiquitous throughout the samples, and not limited to selected grain boundaries.
\end{abstract}

Keywords: Grain boundary segregation; Strontium titanate; EELS; XES; SIMS

\section{Introduction}

Electronic ceramics have become increasingly important as is evidenced by the rapid growth of the electronic ceramics industry, with multi-billion dollar annual sales. The technological importance of these materials depends on the tailoring of the ceramic to obtain the desired electronic properties which can then be exploited to produce a wide array of electronic products. The character of the macroscopic electronic properties of these electronic ceramics, such as varistors, PTCR thermistors, or grain boundary layer capacitors, are often controlled by electrically active grain boundaries. The grain boundary properties can be tailored by varying pro- cessing conditions, such as thermal treatments and furnace environments, as well as by intentional addition of dopants to the material. Consequently, it is critical to understand the nature of the grain boundaries to further refine processing treatments.

Grain boundary segregation plays a critical role in altering the local chemistry and, hence, electronic structure, of the grain boundary region. Kingery $[1,2]$ has discussed the effects of grain boundary structure and characteristics and solute segregation in ceramic materials. Yan et al. [3] also have investigated the nature of solute segregation in ceramics considering elastic, electrostatic, and dipole interactions as the major driving forces for solute segregation in ceramics. Frenkel [4] posited the creation of space 
charge regions about line and planar defects in ionic materials as a consequence of differences in the point defect formation energies of different species. At equilibrium, the difference in formation energies would be manifested as differences in defect populations. In the case of ceramic materials, migration of charged defects to the grain boundaries would result in the formation of space charge regions at the boundaries due to the different concentrations of oppositely charged defect species.

Chiang and Takagi [5] (using XES) and Desu and Payne [6] (using XES, AES and theoretical calculations) have studied the nature of solute segregation in $\mathrm{SrTiO}_{3}$ and $\mathrm{BaTiO}_{3}$ acceptor- and donor-doped samples which were sintered in air or reducing atmospheres. Both groups observed acceptor segregation to the grain boundaries, however Desu and Payne also observed appreciable donor segregation to the grain boundaries while Chiang and Takagi did not. Both groups also observed host cation non-stoichiometry across the interface.

In this paper, we present the results of our investigations of acceptor and donor segregation in both polycrystals and bicrystals of $\mathrm{SrTiO}_{3}$. We have applied a number of analytical electron- and ion-beam techniques, to understand the chemical segregation behavior at grain boundaries in doped $\mathrm{SrTiO}_{3}$. X-ray Emission Spectroscopy (XES) and Electron Energy Loss Spectroscopy (EELS) analysis of grain boundaries were conducted to determine whether there is acceptor and donor solute segregation and, furthermore, what form such a segregation profile would possess. Secondary Ion Mass Spectroscopy (SIMS) [7] was also employed to ensure that the analytical microscopy results were representative of the sample and not simply limited to a few "choice" grain boundaries.

\section{Experimental}

The Fe doped strontium titanate polycrystalline specimen used in this study were prepared by standard mixed oxide techniques and sintered in a flowing oxygen furnace producing a fine $(\leq 2.5 \mu \mathrm{m})$ grained material [8]. The nominal composition of the Fe doped $\mathrm{SrTiO}_{3}$ is $\mathrm{Sr}_{1.00} \mathrm{Ti}_{1.008} \mathrm{Fe}_{0.002} \mathrm{O}_{3}$. In addition to the bulk Fe dopant, a grain boundary specific dopant, $\mathrm{Mn}$, was added via a second firing treat- ment to some of the Fe doped specimen.

Donor ( $\mathrm{Nb}$ )-doped bicrystals of $\mathrm{SrTiO}_{3}$ were obtained from a commercial vendor (Shinkosha Co. Ltd., Japan ). These bicrystals are grown from the melt from oriented seed crystals. The bulk doping level of $\mathrm{Nb}$ is $0.5 \mathrm{wt} . \%$. Through electron diffraction techniques, the misorientation between the grains of this bicrystal was determined to conform to a so called $\Sigma=5$ pure tilt $\left(36.8^{\circ}\right.$ tilt about a common [001] tilt axis) grain boundary. High resolution electron microscopy revealed the boundary to be symmetrical, with a common $\{310\}$ grain boundary plane

Self-supported TEM samples were prepared from these specimen by first ultrasonically drilling $3 \mathrm{~mm}$ discs. These discs were then mechanically thinned and then dimpled to a thickness of $\sim 10 \mu \mathrm{m}$. Lastly, the dimpled discs were milled with $\mathrm{Ar}^{+}$ions in a Gatan Duomill ion-beam thinner at liquid nitrogen temperature (to reduce ion beam damage) until perforation.

All transmission electron microscopy of the samples was carried out in the Hitachi HF-2000 atomic resolution analytical electron microscope (ARAEM). This microscope is equipped with a cold field emission gun (cFEG) and can be operated at accelerating voltages of up to $200 \mathrm{kV}$. The microscope is fitted with an Oxford ultra-thin window energy dispersive X-ray spectrometer for XES acquisition which allows detection of light elements down to carbon. It is also equipped with a Gatan 666 parallel electron energy loss spectrometer (PEELS) for electron energy loss spectra acquisition with an energy resolution of less than $0.5 \mathrm{eV}$ (full width at half maximum for zero loss peak). All EELS spectra were acquired on a Macintosh IIfx computer running Gatan EL/P software. In order to reduce the effects of carbon contamination during microanalysis work, a liquid nitrogen TEM specimen holder was employed for all PEELS and XES studies. SIMS experiments were conducted on the University of Chicago SIMS [7].

The spatial resolution, $R$, of the XES experiments was considered. $R$ is limited by the probe size, $d$, of the electron beam impinging on the specimen surface and the beam broadening, $b$, caused by elastically scattered electrons traveling through the sample, and is given by [9]

$R=\sqrt{d^{2}+b^{2}}$.

The probe size, $d$, was estimated to be $2.5 \mathrm{~nm}$ at full- 
width-at-tenth-maximum (FWTM). The beam broadening, $b$, was estimated using a Monte Carlo simulation program developed by Joy [10]. An EEL low loss spectrum was acquired to calculate the thickness of the sample, which was conservatively estimated at $500 \AA$. Utilizing the single scattering simulation, a $90 \%$ beam broadening estimate of $1.2 \mathrm{~nm}$ was obtained for strontium titanate based on the trajectories of 30000 electrons. Based on these estimates, the spatial resolution was calculated to be $\sim 2.8$ $\mathrm{nm}(d \approx 2.5 \mathrm{~nm}, b \approx 1.2 \mathrm{~nm})$.

EELS analysis helps to avoid many of the shortcomings associated with XES studies. The increased intensity of the signal in EELS allows much shorter collection times when compared with XES which helps to reduce contamination problems as well as beam and sample drift during microanalysis. Acquisition times for the individual spectra varied from less than a second for the low loss region up to a maximum of $12.8 \mathrm{~s}$ for the core loss region with eight of these spectra acquired sequentially and added to produce a final spectrum. The X-ray emission spectra, on the other hand, took as long as 5-7 min to collect. Also, peak overlap, often a problem for elements of similar atomic number in XES, is avoided in EELS due to superior energy resolution with access to many edges in addition to the standard $K$ and $L$ lines used in XES. Finally, core loss EELS does not suffer from delocalization of the signal (since delocalization is inversely proportional to the energy loss [9]) and hence the spatial resolution is essentially limited by the probe size. The spectra were acquired in the diffraction mode which allows focusing of the transmitted spot on the collection entrance aperture. The collection semi-angle, $\beta$, was $7.5 \mathrm{mrad}$ for all experiments.

\section{Results and discussion}

\subsection{SIMS analysis}

The SIMS analysis indicated uniform presence of $\mathrm{Sr}$ and $\mathrm{Ti}$ throughout most of the sample in both $\mathrm{Fe}$ and $\mathrm{Fe} / \mathrm{Mn}$ doped $\mathrm{SrTiO}_{3}$ as would be expected from the $\mathrm{SrTiO}_{3}$ matrix. However, inhomogeneities are observed in both specimens through the presence of bulk second phase regions. These second phase re- gions are depleted in $\mathrm{Sr}$ in the case $\mathrm{Fe} / \mathrm{Mn}$ doped specimen, as can be seen from Fig. 1, containing a series of SIMS micrographs of a single $40 \times 40 \mu \mathrm{m}^{2}$ region. Within these second phase regions, the presence of $\mathrm{Fe}$ and $\mathrm{Mn}$ is also enhanced, as can be seen from the $\mathrm{Fe}$ and $\mathrm{Mn}$ ion maps in Fig. 1. Scans from the Fe-doped sample also revealed bulk second phases which are $\mathrm{Ti}$ and $\mathrm{Fe}$ rich but depleted in $\mathrm{Sr}$.

These second phase regions were encountered in the course of microanalysis work in the TEM studies as well. Fig. 2 shows an X-ray emission spectrum from the $\mathrm{Fe} / \mathrm{Mn}$ doped sample. The spectrum was collected from the bulk of a second phase grain; it clearly shows $\mathrm{Mn}$ enrichment and $\mathrm{Sr}$ depletion. Interestingly, there is no evidence of enhanced segregation of Fe or Mn near these second phase regions. XES from grain boundaries adjacent to the $\mathrm{Mn}$ rich grains did not show any enhancement of the Mn signal, as one might expect due to the $\mathrm{Mn}$ reservoirs offered by the second phase grains. We therefore presume that these second phases are stoichiometric manganese titanate grains, with no excess Mn. The SIMS micrographs in Fig. 1 depict this as well; in the regions immediately about the second phases, there is no indication of increased dopant concentration, either in the bulk or at the grain boundaries.

The origin of these second phases is most definitely due to the ceramic processing techniques employed to fabricate these samples. Inhomogeneities in the starting ball-milled powders could lead to iron rich regions which could form iron titanate compounds. Moreover, the mixing of $\mathrm{MnO}$ and $\mathrm{SrTiO}_{3}$ for the second firing treatment could also produce manganese titanate compounds. One would expect these phases to be present near the surfaces of the original sintered ceramics due to bulk diffusion considerations.

In addition to the exposure of second phase regions in the doped strontium titanate bulk samples, the SIMS studies also depicted preferential Mn segregation at the grain boundaries. The $\mathrm{Mn}$ ion map in Fig. 3 does show an inhomogeneous distribution of Mn throughout the sample with enrichment at the grain boundaries. Such preferential segregation is to be expected as the grain boundaries offer a rapid diffusion path for the dopant material. Note that the width of the Mn segregation layer is limited to the resolution of the SIMS probe, which is roughly $50 \mathrm{~nm}$. 


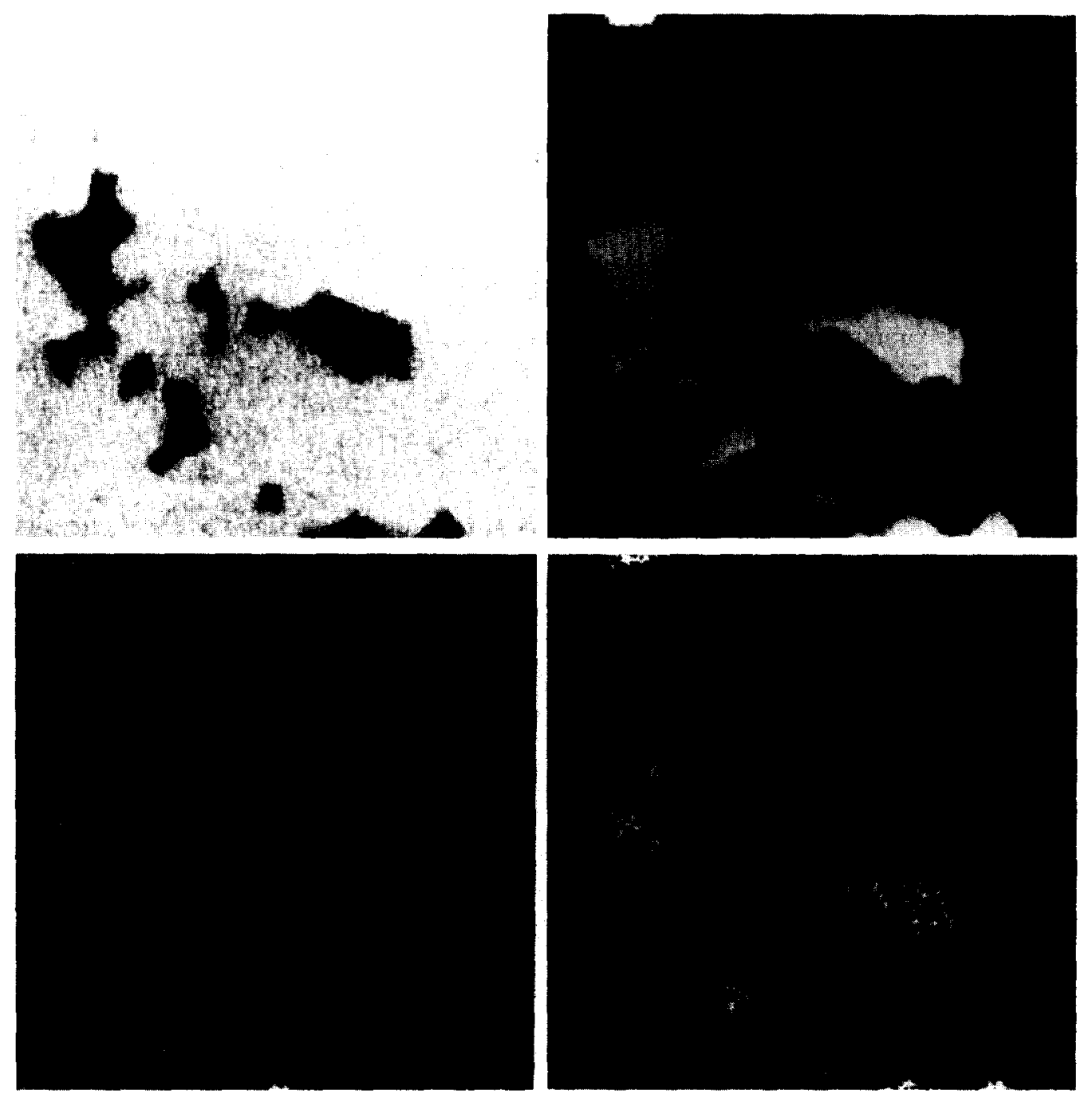

Fig. 1. SIMS micrographs of a representative region of the $\mathrm{Fe} / \mathrm{Mn}$ doped $\mathrm{SrTiO}_{3}$ polycrystal. Clockwise from top left corner: $\mathrm{Sr}$, $\mathrm{Ti}$, $\mathrm{Mn}$, and $\mathrm{Fe}$ ion maps. Images are taken from a $40 \times 40 \mu \mathrm{m}^{2}$ area and indicate the presence of second phases.

Aside from the second phase regions, the $\mathrm{Mn}$ ion signal is limited to one pixel in width.

The evidence of Fe segregation was not as clearly convincing as that of $\mathrm{Mn}$ with the SIMS analysis. The Fe ion map in Fig. 4 hints at a heterogeneous distribution of Fe between the grain bulk and grain boundaries; however, the results are hardly conclusive. The segregation profiles suggested by the SIMS analysis for these acceptor doped samples were at the limits of the SIMS technology and therefore necessitate the use of other analytical techniques for further study.

\subsection{XES microanalysis}

XES microchemical analysis of the grain boundaries proved challenging due to the small amounts of dopant material added to the material. In all cases, dopant scgregation levels fell off to the background levels within 5 to $10 \mathrm{~nm}$ from the grain boundaries. There was no evidence of second phases or amorphous phases decorating the grain boundaries in either the Fe-doped or Fe/Mn-doped polycrystalline samples. Grain boundaries chosen for XES profiling were all oriented parallel to the EDS detector. A typ- 


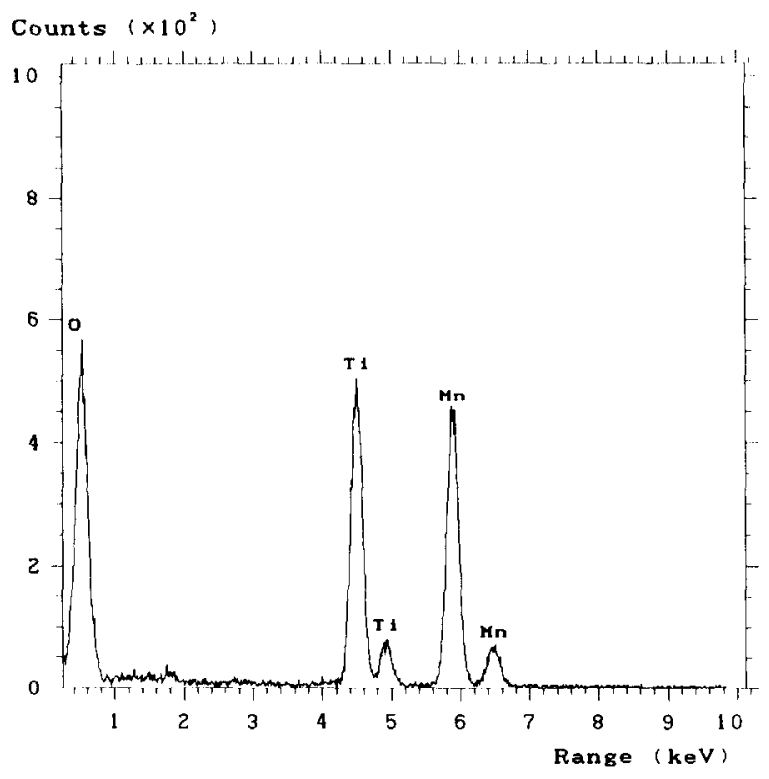

Fig. 2. X-ray emission spectrum from a second phase grain in $\mathrm{Fe} /$ $\mathrm{Mn}$ doped $\mathrm{SrTiO}_{3}$ polycrystal.

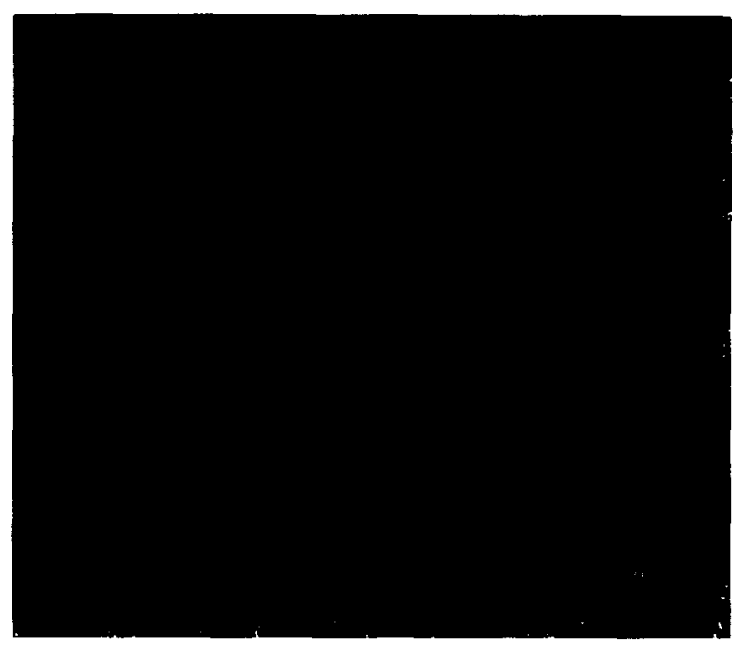

Fig. 3. $\mathrm{Mn}$ ion SIMS micrographs of the $\mathrm{Fe} / \mathrm{Mn}$ doped $\mathrm{SrTiO}_{3}$ polycrystal. Grain boundary segregation of $\mathrm{Mn}$ is evident in this $10 \times 10 \mu \mathrm{m}^{2}$ region.

ical spectrum from a grain boundary in the Fe-doped sample is shown in Fig. 5a while the corresponding spectrum collected $5 \mathrm{~nm}$ from this boundary is given in Fig. 5b. These spectra are representative of all of the boundaries examined. The grain boundaries show iron segregation with a segregation width of less than $10 \mathrm{~nm}$. These results are consistent with those obtained by Chiang and Takagi [5]. The bulk Fe levels

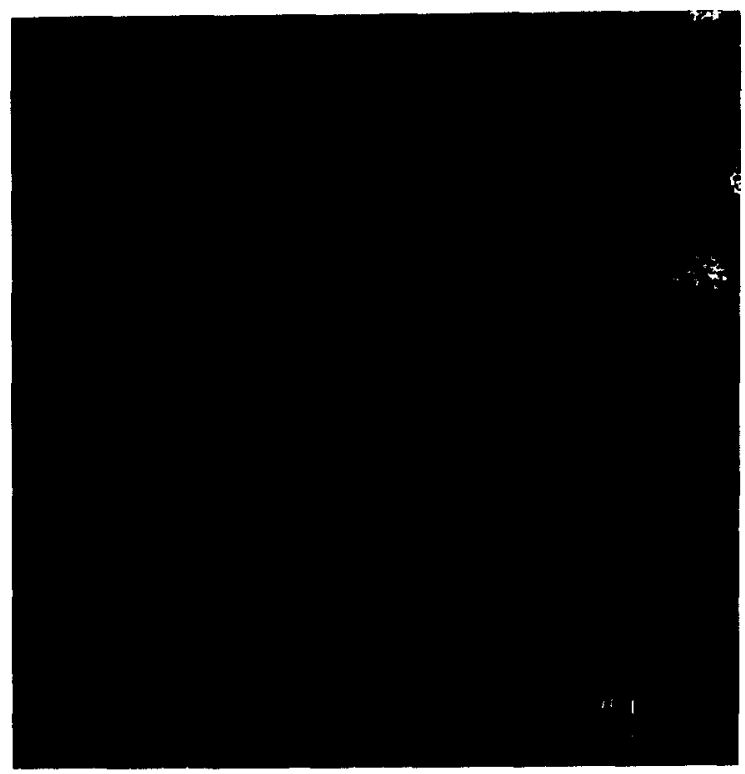

Fig. 4. Fe ion SIMS micrograph of the $\mathrm{Fe}$ doped $\mathrm{SrTiO}_{3}$ polycrystal.

are essentially too low to be detected above background due to the low dopant concentration, despite the fact that the Fe was added as a bulk dopant. Furthermore, the segregation of $\mathrm{Fe}$ to the boundaries as well as the presence of Fe rich bulk second phases (as seen with SIMS) would further decrease the concentration of $\mathrm{Fe}$ in the bulk of $\mathrm{SrTiO}_{3}$ grains.

Fig. 6a shows a composite plot of the Fe/Ti peak intensity ratio for all of the grain boundaries studied in the Fe-doped sample. The horizontal error bars represent the effects of beam broadening as well as an estimated $10 \%$ error in probe positioning. The vertical error bars reflect the $90 \%$ confidence level for Student $t$-analysis of the data. Peak counts were arrived at by integrating the signal over the full width half maximum (FWHM) region of each peak and then subtracting the background. The background was approximated by summing the counts in the same energy width as the peak FWHM on either side of the peak. There is a clear trend of Fe segregation to the boundaries, although there is a spread in the amounts of segregation seen at individual boundaries. No statistically significant variations in the $\mathrm{Ti} / \mathrm{Sr}$ (Fig. 6b) peak intensity ratio could be detected between the bulk and the grain boundary.

Similarly for the Fe/Mn doped sample, $\mathrm{Mn}$ is confined to the grain boundaries or bulk second phases. 

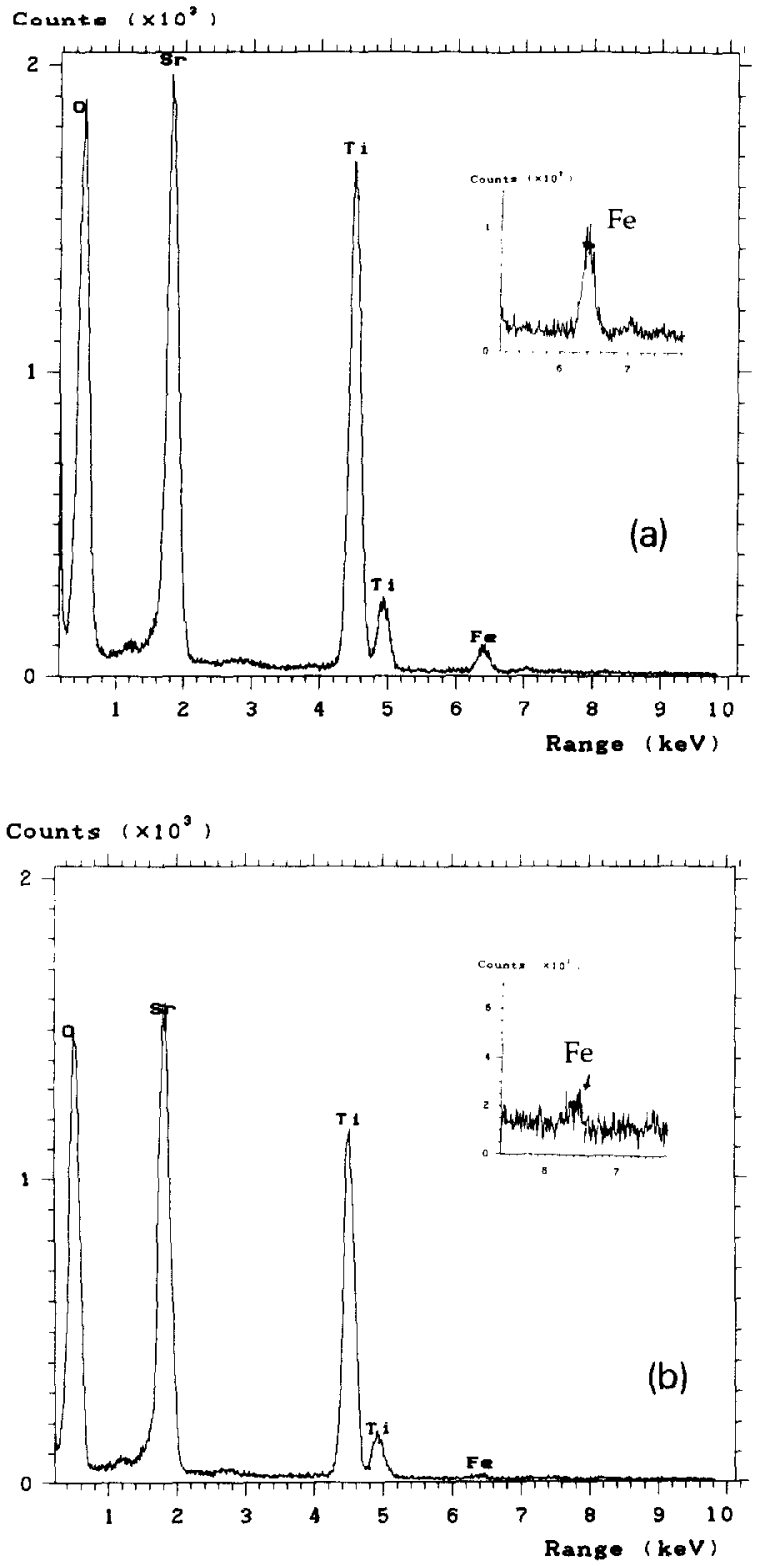

Fig. 5. (a) X-ray emission spectrum from a grain boundary of the $\mathrm{Fe}$ doped $\mathrm{SrTiO}_{3}$ polycrystal; (b) X-ray emission spectrum 5 $\mathrm{nm}$ away from the same boundary as of (a).

The spectra shown in Fig. 7a and $\mathbf{b}$ are typical of the segregation behavior seen for $\mathrm{Mn}$ in this sample. Again, the bulk spectra was taken $5 \mathrm{~nm}$ away from the interface and the $\mathrm{Mn}$ signal has essentially dropped to background levels at this distance. The $\mathrm{Ar}$ peak present in this spectra is an artifact from ion beam thinning and was traced to the specific IBT
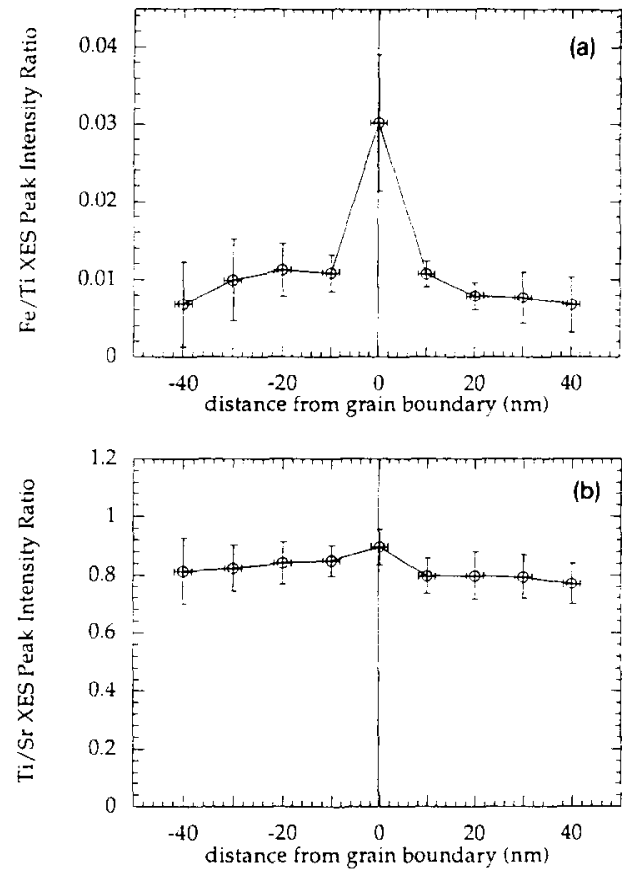

Fig. 6. (a) Fe/Ti XES segregation profile from all the grain boundaries studied in the $\mathrm{Fe}$ doped $\mathrm{SrTiO}_{3}$ polycrystal. Horizontal error bars include $10 \%$ positioning crror and beam broadening estimates, while vertical error bars are for $90 \%$ Student $t$ analysis confidence level; (b) $\mathrm{Ti} / \mathrm{Sr}$ XES segregation profile from all the grain boundaries studied in the $\mathrm{Fe}$ doped $\mathrm{SrTiO}_{3}$ polycrystal. Horizontal error bars include $10 \%$ positioning error and beam broadening estimates, while vertical error bars are for $90 \%$ Student $t$-analysis confidence level.

routine used prior to that particular microscopy session.

Again, the composite plots for all the grain boundaries studied in the $\mathrm{Fe} / \mathrm{Mn}$ doped $\mathrm{SrTiO}_{3}$ are given in Fig. $8 \mathrm{a}$ and $\mathrm{b}$. The results are similar to those obtained for Fe segregation, although the mechanisms involved are different. The $\mathrm{Mn}$ segregation is limited to a region within $10 \mathrm{~nm}$ of the boundary and the intensity varies slightly from boundary to boundary. Unfortunately, the study of Fe segregation in the Mn/ Fe doped samples was hampered by the overlap of the $\mathrm{Mn} \mathrm{K} \beta$ and Fe K $\alpha$ peaks. Furthermore, it is likely that Fe X-ray excitations could lead to fluorescence of $\mathrm{Mn}$ species thus resulting in enhanced Mn excitations and depleted Fe excitations. Nonetheless, the Fe segregation behavior of the $\mathrm{Mn} / \mathrm{Fe}$ doped sample is expected to be similar to that detected in the $\mathrm{Fe}$ 

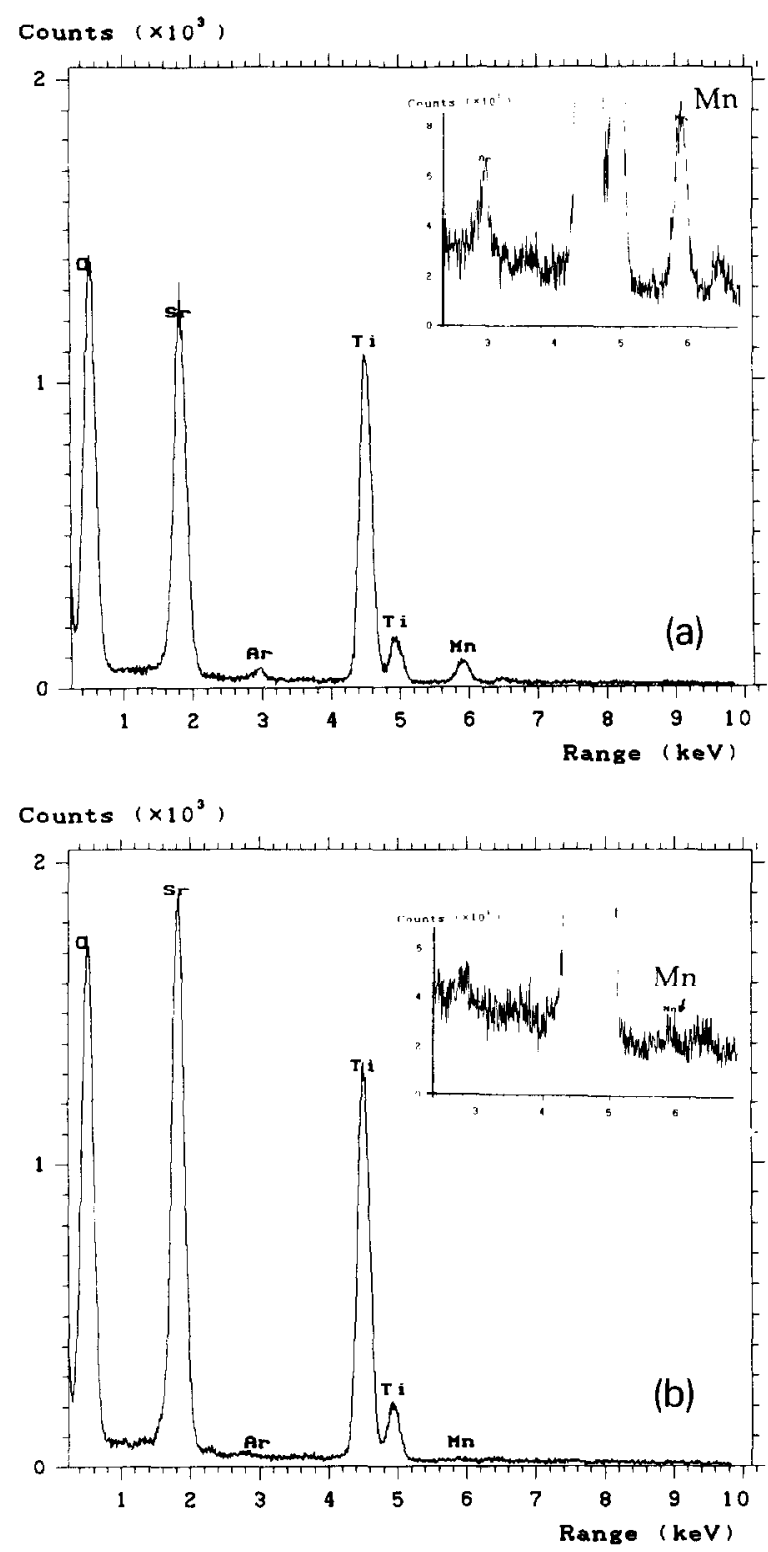

Fig. 7. (a) X-ray emission spectrum from a grain boundary of the $\mathrm{Fe} / \mathrm{Mn}$ doped $\mathrm{SrTiO}_{3}$ polycrystal; (b) X-ray emission spectrum $5 \mathrm{~nm}$ away from the same boundary as of (a).

doped sample as the materials are the same up to the second firing treatment.

The results of the XES profiles across the $\mathrm{Mn} / \mathrm{Fe}$ doped samples agree well with the suggestion of grain boundary segregation hinted at by SIMS. The agreement with the SIMS experiments indicates that the $\mathrm{Mn}$ segregation seen by the AEM microanalysis is not
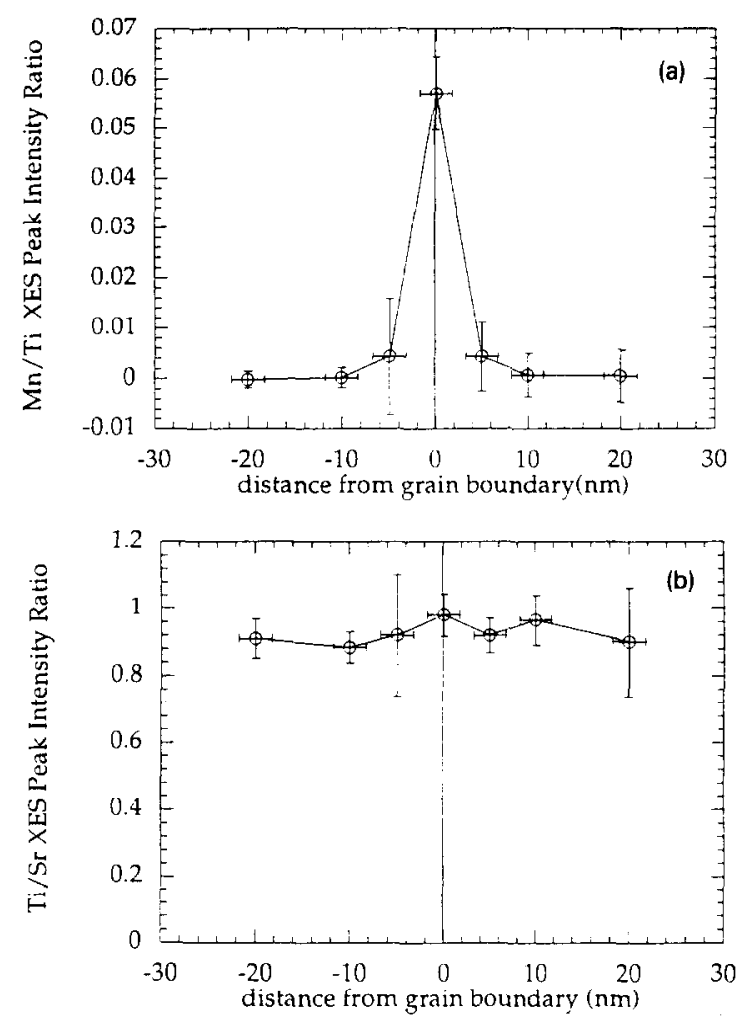

Fig. 8. (a) Mn/Ti XES segregation profile from all the grain boundaries studied in the $\mathrm{Fe} / \mathrm{Mn}$ doped $\mathrm{SrTiO}_{3}$ polycrystal. Horizontal error bars include $10 \%$ positioning error and beam broadening estimates, while vertical error bars are for $90 \%$ Student analysis confidence level; (b) Ti/Sr XES segregation profile from all the grain boundaries studied in the Fe/Mn doped Sr$\mathrm{TiO}_{3}$ polycrystal. Horizontal error bars include $10 \%$ positioning error and beam broadening estimates, while vertical error bars are for $90 \%$ Student $t$-analysis confidence level.

restricted to a few special boundaries, but is indeed a general fealure of the sample. The presence of grain boundary segregation of $\mathrm{Fe}$ was less convincing from the SIMS analysis than that in the AEM. However, this is likely due to the smaller amounts of Fe segregation, which involves diffusion of $\mathrm{Fe}$ ions through the bulk to grain boundaries, than Mn segregation which was carried out via a rapid grain boundary diffusion mechanism.

The effect of a donor dopant was studied by XES in the case of the $\mathrm{Nb}$-doped $\mathrm{SrTiO}_{3}$ bicrystal. The results of the XES investigation for $\mathrm{Nb}$ segregation are not as conclusive as those for acceptor doped solutes. There is a hint of $\mathrm{Nb}$ segregation to the grain boundary, in agreement with the findings of Desu and Payne 
[6]; however the limited number of profiles acquired leave the results less conclusive. Spectra were collected from the bulk and the grain boundary using both a spot probe (as with the XES analysis of the acceptor doped $\mathrm{SrTiO}_{3}$ ) and also a "line" probe along the grain boundary. The raw spectra collected from the boundary and from the bulk are compared in Fig.
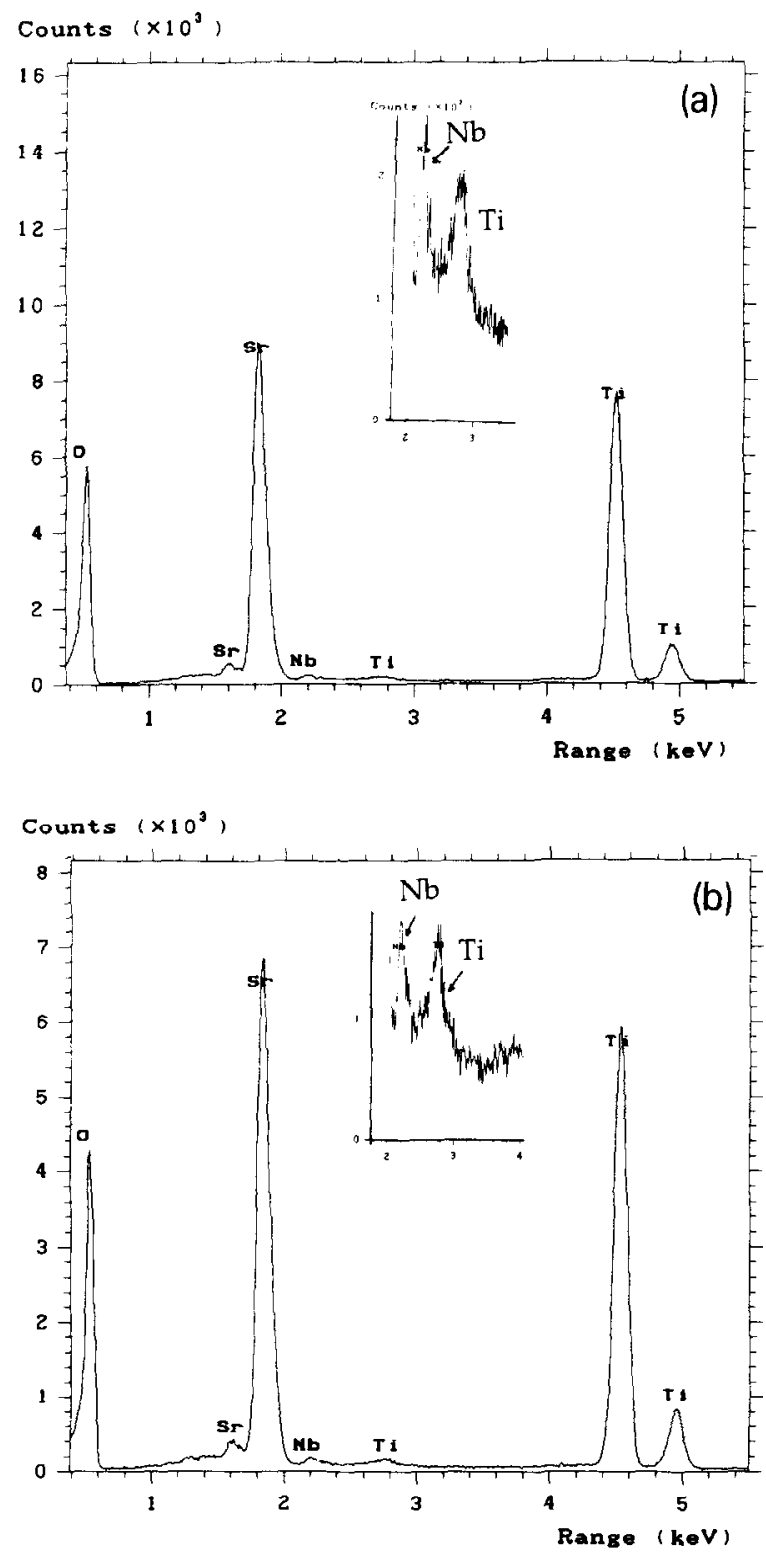

Fig. 9. (a) X-ray emission spectrum from a grain boundary of the $0.05 \mathrm{wt} \% \mathrm{Nb}$ doped $\mathrm{SrTiO}_{3}$ bicrystal; (b) X-ray emission spectrum $5 \mathrm{~nm}$ away from the same boundary as of (a).
$9 \mathrm{a}$ and $\mathrm{b}$ while the results of the profiles are shown in Fig. 10a and b. The vertical error bars in Fig. 10 are those for a "one $\sigma$ " ( $67 \%$ confidence level) deviation. The enhancement of the $\mathrm{Nb} / \mathrm{Ti}$ peak intensity ratio scen to the right of the interface is likely an artifact due to poor alignment of the grain boundary with the detector. The grain boundary was oriented such that it was almost perpendicular to the detector with the spectra composing the right hand side of the plot in Fig. 10 coming from the crystal that was opposite the detector. Consequently, X-rays from this crystal would have traveled through the interface on their way to the detector, leading to enhancement of the $\mathrm{Nb}$ signal for this crystal. As with the acceptor-
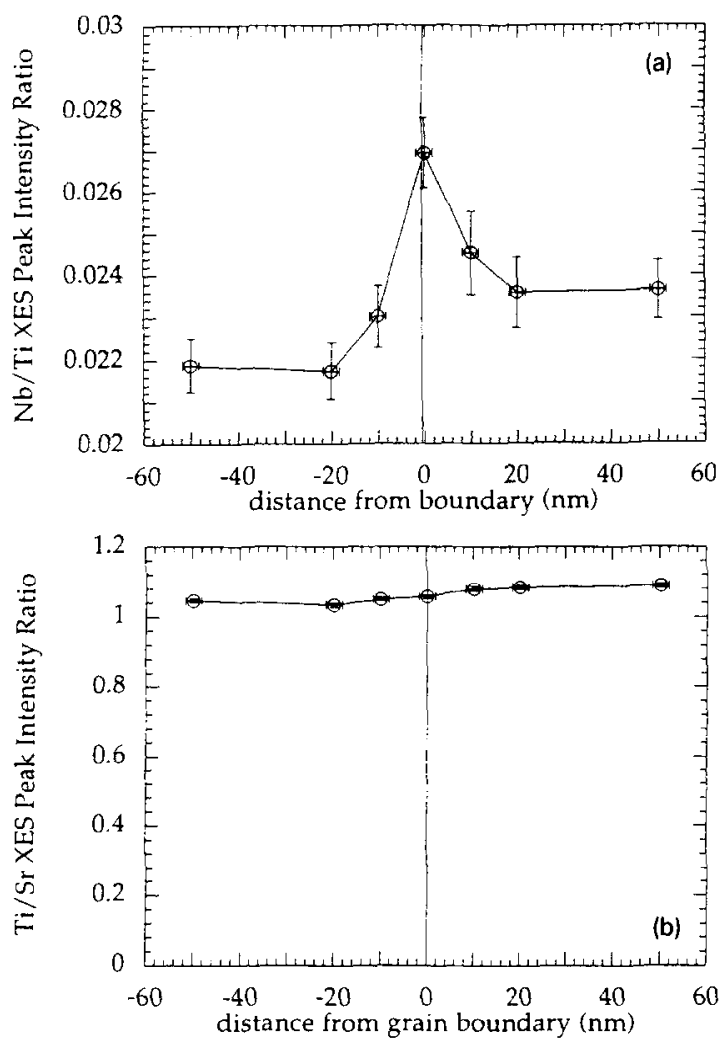

Fig. 10. (a) Nb/Ti XES segregation profile for the $0.05 \mathrm{wt} \% \mathrm{Nb}$ doped $\mathrm{SrTiO}_{3}$ bicrystal. Horizontal error bars include $10 \%$ positioning error and beam broadening estimates, while vertical error bars are for a $67 \%$ confidence level; (b) Ti/Sr XES segregation profile for the $0.05 \mathrm{wt} \% \mathrm{Nb}$ doped $\mathrm{SrTiO}_{3}$ bicrystal. Horizontal error bars include $10 \%$ positioning error and beam broadening estimates, while vertical error bars are for a $67 \%$ confidence level. 
doped samples, there was no evidence of a second phase at the grain boundary in the $\mathrm{Nb}$ doped bicrystal.

\subsection{EELS microanalysis}

To perform core loss EELS, a line probe was positioncd along the grain boundary and then stcpped away from the interface. The effect of the line probe is to average the signal along the boundary. The results of EELS profiling across the grain boundary of $\mathrm{Mn} / \mathrm{Fe}$ doped sample are given in Fig. 11. Again, the results of the XES and SIMS studies are confirmed by the spike in the $\mathrm{Mn} / \mathrm{Ti}$ edge intensity ratio at the interface. The raw spectra (corrected for channel to channel gain variation and dark current) from a grain boundary and from a bulk position $5 \mathrm{~nm}$ away from the grain boundary are displayed in Fig. 12. The energy dispersion is $0.3 \mathrm{eV} /$ channel. The presence of the $\mathrm{Mn} \mathrm{L}$ edge is clearly visible in the grain boundary spectrum; however, it is absent in the bulk spectrum. Note that the Fe edge, which should be found at 708 $\mathrm{eV}$, is not detected either in the bulk or at the boundary. Again, the Fe signal is apparently too weak to be detected above the background of the spectra. Some variation in the $\mathrm{Ti} \mathrm{L}_{1}$ edge and in the $\mathrm{Ti} \mathrm{L}_{2 / 3}$ near edge structure is also apparent between the bulk and grain boundary spectra. These variations may be due to local changes in the bonding of $\mathrm{Ti}$ at the grain

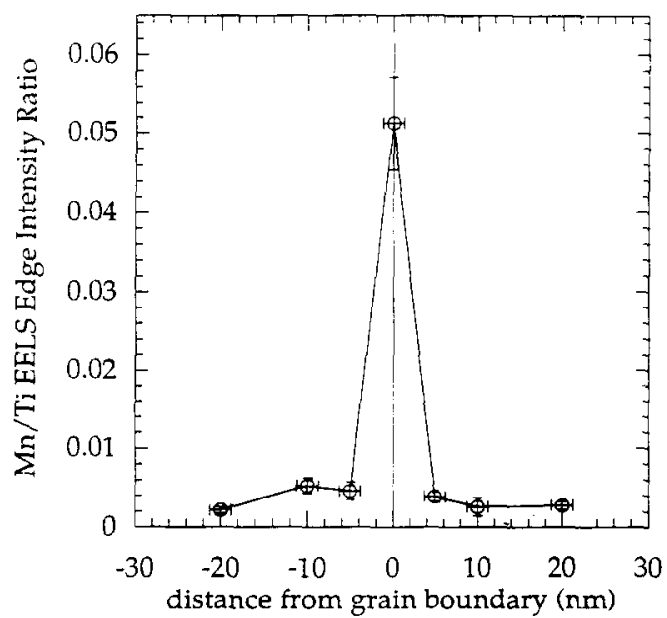

Fig. 11. Segregation profile in the Fe/Mn doped polycrystal using EELS core loss. Horizontal error bars include $10 \%$ positioning error and probe size while vertical error bars are $90 \%$ confidence level.
Comparison of core loss EELS at gb and bulk

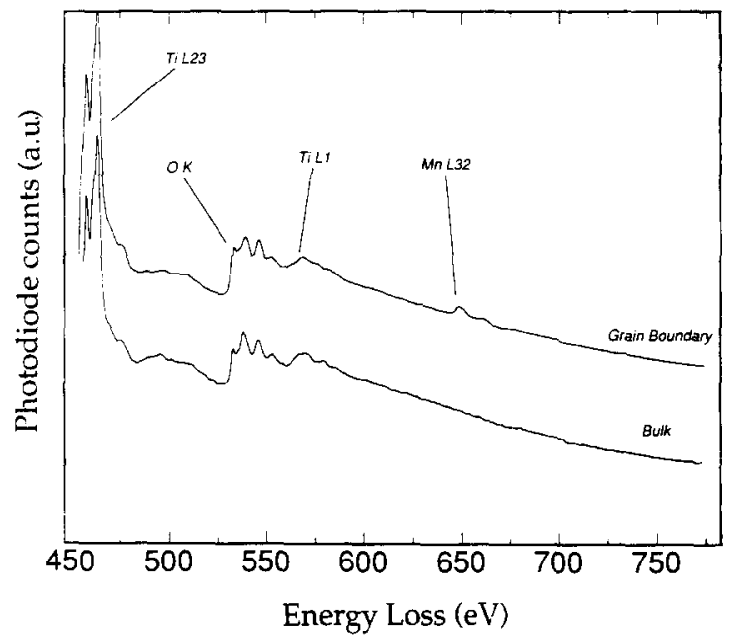

Fig. 12. Comparison of EELS core loss at the grain boundary and the bulk in the $\mathrm{Fe} / \mathrm{Mn}$ doped $\mathrm{SrTiO}_{3}$ polycrystalline sample.

boundaries. Although we have yet to fully explore the nature of these variations, they are indicative of the wealth of information offered by EELS.

\section{Conclusions}

The results of this study further support the significance of grain boundary segregation in perovskite materials. XES and EELS studies of segregation profiles for $\mathrm{Fe}$ and $\mathrm{Mn}$ in acceptor-doped strontium titanate polycrystals show a pronounced segregation to the grain boundaries with very steep gradients away from the boundary. Fe and Mn segregation is limited to within a $5 \mathrm{~nm}$ distance about the boundary, or to the limits of step size in this experiment, without the presence of any second phases at the boundary. Moreover, XES studies do not indicate any host cation non-stoichiometry at the interface. The generality of the XES and EELS segregation profiles is confirmed by SIMS analysis of the acceptor doped samples which indicated $\mathrm{Mn}$ segregation to the grain boundaries and hint at Fe segregation as well. The chemical microanalysis of the interfaces of donordoped $\mathrm{SrTiO}_{3}$ shows slight segregation of the $\mathrm{Nb}$ donor dopant species for a lightly doped $(0.5 \mathrm{wt} \%)$ strontium titanate bicrystal. Such measurements demonstrated the utility of applying a battery of microanalytic techniques in studying relevant materials 
issues and the consistency of the results presents a coherent picture of the nature of the grain boundaries in doped strontium titanate.

\section{Acknowledgment}

This work is supported by the U.S. Department of Energy-Basic Energy Sciences, Grant No: DE-FG0292ER45475. We thank Prof. Riccardo Levi-Setti for permission to use the U.C. SIMS. Partial travel support to attend the Symposium on Interfaces in Ionic Materials, Schloss Ringberg, from the Office of $\mathrm{Na}$ val Research by Dr. S.G. Fishman is gratefully acknowledged.

\section{References}

[1] W.D. Kingery, J. Am. Ceram. Soc. 57 (1974) 1.

[2] W.D. Kingery, J. Am. Ceram. Soc. 57 (1974) 74.

[3] M.F. Yan, R.M. Cannon and H.K. Bowen, in: Advances in Ceramics, Vol. 6, eds. M.F. Yan and A.H. Heuer (The American Ceramic Society, Columbus, $\mathrm{OH}, 1983$ ).

[4] J. Frenkel, Kinetic Theory of Liquids (Oxford University Press, New York, 1946).

[5] Y.M. Chiang and T. Takagi, J. Am. Ceram. Soc. 73 (1990) 3278.

[6] S.B. Desu and D.A. Payne, J. Am. Ceram. Soc. 73 (1990) 3391.

[7] R. Levi-Setti et al., Scanning Microsc. 7 (1994) 1161.

[8] M. Vollmann and R. Waser, preprint of the Proceedings article.

[9] D.C. Joy, A. Romig $\mathbf{J}_{\mathrm{r}}$, and J.I. Goldstein, Principles of Analytical Electron Microscopy (Plenum Press, New York, 1986).

[10] D.C. Joy, Monte Carlo simulations of electron beam-solid interactions (University of Tennessee, 1994). 